\title{
The potential of bioorthogonal chemistry for correlative light and electron microscopy: a call to arms
}

\author{
Daphne M. van Elsland ${ }^{1,2} \cdot$ Erik Bos $^{3} \cdot$ Herman S. Overkleeft ${ }^{1,2}$ • \\ Abraham J. Koster ${ }^{3}$ - Sander I. van Kasteren ${ }^{1,2}$
}

Received: 2 December 2014 / Accepted: 27 April 2015 / Published online: 26 May 2015

(C) The Author(s) 2015. This article is published with open access at Springerlink.com

\begin{abstract}
With correlative light and electron microscopy (CLEM), the ultrastructural cellular location of a biomolecule of interest can be determined using a combination of light microscopy (LM) and electron microscopy (EM). In many cases, the application of CLEM requires the use of markers that need to be attached to a biomolecule of interest to allow its identification and localization. Here, we review the potential of bioorthogonal chemistry to introduce such markers for CLEM.
\end{abstract}

Keywords Bioorthogonal chemistry · Click chemistry . Electron microscopy $\cdot$ CLEM

\section{Introduction}

Correlative light and electron microscopy (CLEM) is an imaging technique that combines the virtues of light microscopy (LM) with those of electron microscopy (EM). With this technique, specific molecular and cellular structures in a cell can be identified with LM after which ultrastructural information

Abraham J. Koster and Sander I. van Kasteren share the corresponding authorship.

Abraham J. Koster

a.j.koster@lumc.nl

$\triangle$ Sander I. van Kasteren

s.i.van.kasteren@chem.leidenuniv.nl

1 Division of Bio-organic Synthesis, Leiden Institute of Chemistry, Leiden University, Leiden, The Netherlands

2 Institute for Chemical Immunology, Leiden, The Netherlands

3 Molecular Cell Biology, Leiden University Medical Center, Leiden, The Netherlands about its subcellular location and context can be obtained. CLEM studies that involve fluorescence microscopy may benefit from fluorescent markers that can be attached to molecules of interest to allow their identification and localization. To date, most readily this has been done by fluorescent fusion proteins, by fluorescent antibody labelling or by the chemical modification of a protein with a fluorescent detection group [1-3]. As well as these fluorescent detection moieties, structures must be present in the CLEM sample that are both EM and LM detectable in order to correlate (overlay) the LM image with the EM image. Examples of such EM/LM detectable structures are fluorescently labelled cellular structures that are suitable to be identified by EM through their distinct morphology (e.g. stained nuclei) or fluorescently labelled electron-dense particles (e.g. fluorescent microspheres) [1-4].

The above-mentioned labelling approaches have been very successfully applied to CLEM imaging of specific proteins in their cellular context. However, they carry some limitations. First, the use of fluorescent fusion proteins requires genetic manipulation of the cell, which can be difficult and can affect the function of the protein of interest [5]. An alternative to genetic manipulation is antibody labelling. However, for specimens prepared for CLEM, antibody labelling is an elaborate process of which the success rate is notoriously low due to lack of availability of functional antibodies [6]. Finally, all these labelling approaches do not readily allow imaging of non-templated biomolecules, such as glycans and lipids.

Bioorthogonal chemistry is a powerful new labelling tool that circumvents the disadvantages mentioned above and allows for the imaging of a wide range of biomolecules. Its mechanism [7] relies on the introduction of a small abiotic chemical group (one that is non-reactive with other chemical functionality found in the cell) into a biomolecule of interest which can be specifically reacted with a detection moiety using a so-called bioorthogonal chemical reaction, a reaction of the 
A)

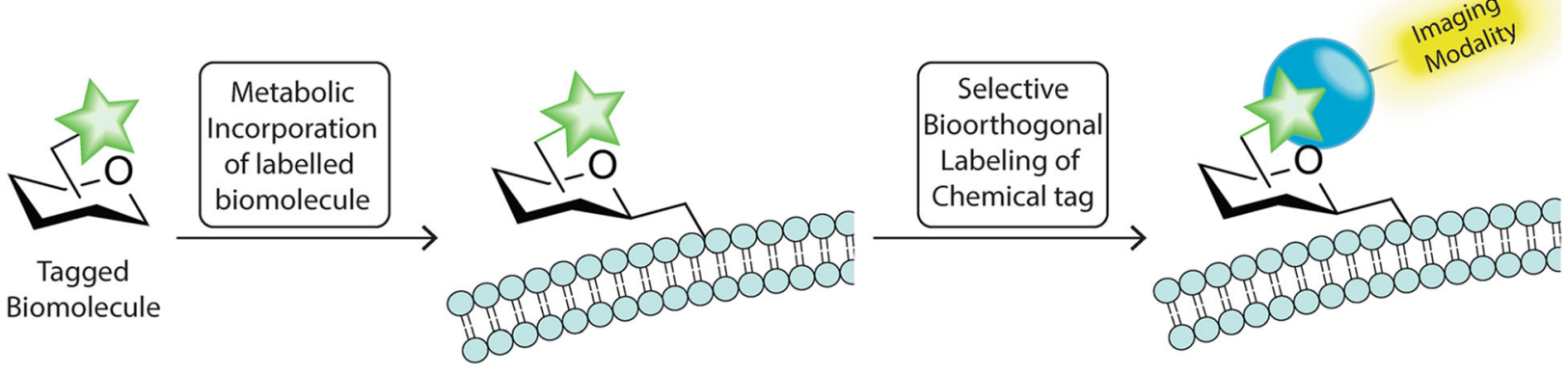

B)
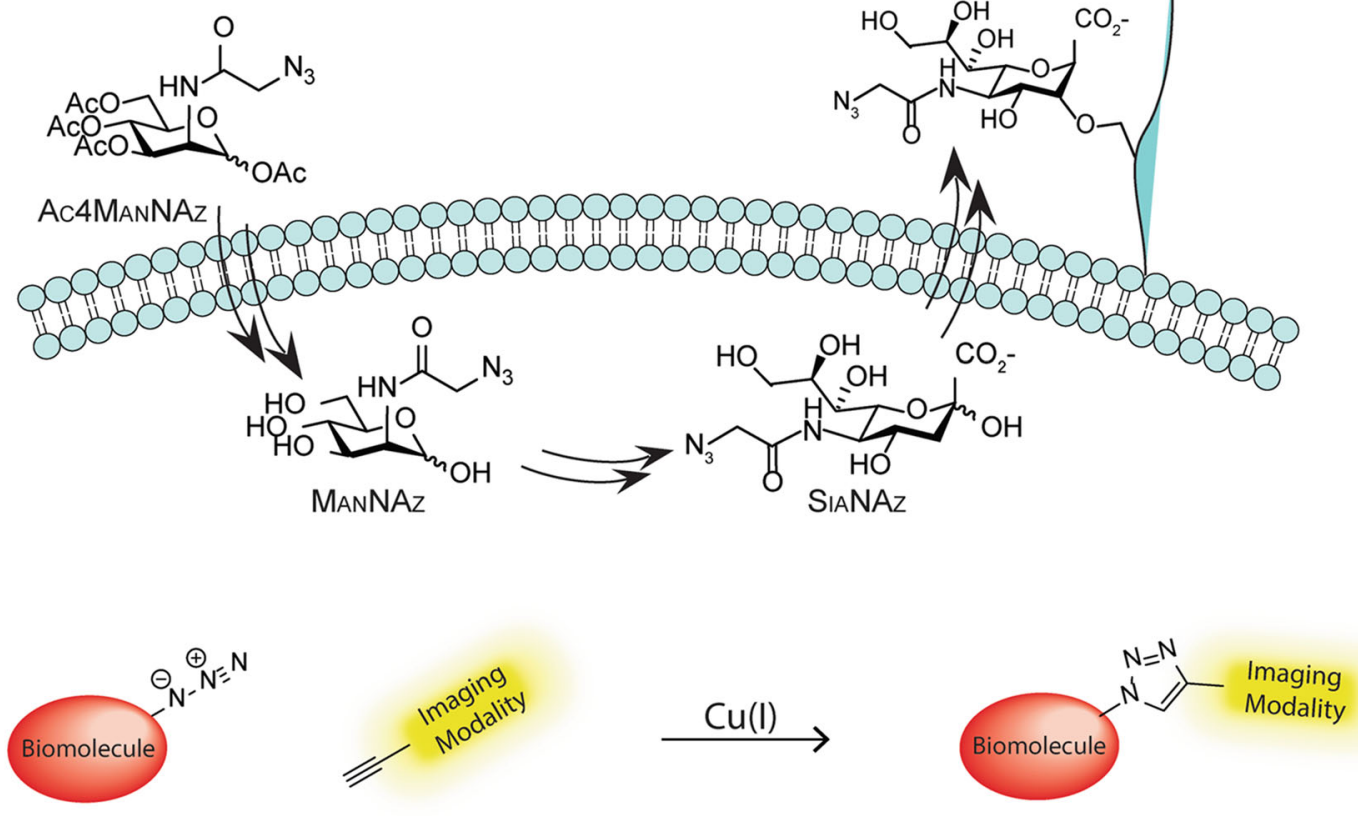

D)
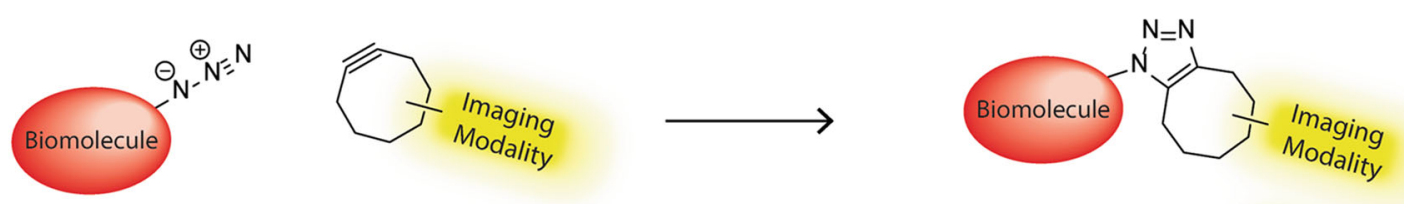

E)
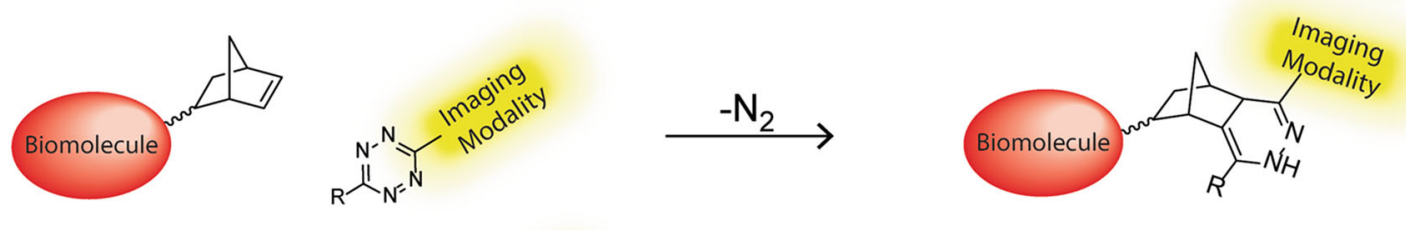

\section{F)}
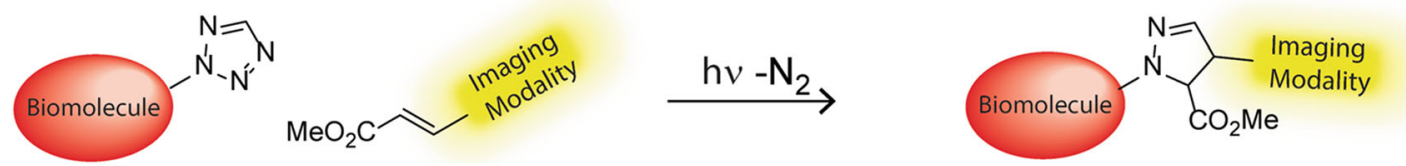

Fig. 1 Bioorthogonal chemistry for imaging. a General approach: a biologically inert group is incorporated into a biomolecule class in a living cell and selectively visualized using chemistry specific for this bioorthogonal group. b An azide-labelled $N$-acetylmannosamine analogue is converted to CMP-sialic acid in vivo. Azido-sialic is then

incorporated into the nascent glycoproteins. c-f Recently applied bioorthogonal reactions for imaging, such as the copper-catalysed Huisgen cycloaddition (c) [25], the strain-promoted cycloaddition (d) [26], the inverse electron-demand Diels-Alder cycloaddition (e) [27], or the photoclick reaction (f) [28]

tag with a detectable group that is essentially background-free in biological systems (Fig. 1a) [8]. As this labelling strategy

makes use of a small chemical group to tag a biomolecule of interest, it minimally interferes with the structure of the labelled 
biomolecule and as such minimally affects cellular biochemistry [9]. Since the initial development of the Staudinger-Bertozzi ligation, bioorthogonal labelling chemistry has evolved rapidly. Currently, a wide-ranging chemical toolkit is available of both tags for incorporation into biomolecules and reactions for subsequent labelling of these tags [10]. The choice of tag and modification chemistry can therefore be optimized and tailored for the specific biological hypothesis [7].

The introduction of a bioorthogonal tag into a biomolecule of interest occurs most readily by the metabolic incorporation of a tagged biomolecule building block. One of the approaches that exemplify the metabolic incorporation strategy was reported by Saxon et al. [11]. They synthesized a cell-permeable azide-tagged $N$-acetylmannosamine analogue ( $\left.\mathrm{Ac}_{4} \mathrm{ManNAz}\right)$ which was administered to mammalian cells during cell culture [12]. Inside these cells, the acetyl groups were removed from $\mathrm{Ac}_{4} \mathrm{ManNAz}$ after which it was passed on to the steps of the sialic acid biosynthetic pathway where it was converted to SiaNAz. After conversion to the nucleotide sugar CMPSiaNAz, SiaNAz is incorporated into various glycoconjugates by sialyltransferase enzymes. With this approach, Saxon et al. produced cells containing azide-tagged sialoglycans and visualized these using the Staudinger-Bertozzi reaction (Fig. 1b).

Since this first inception, the applications and classes of biomolecules that can be labelled with this approach have expanded rapidly. For instance, Salic and Mitchinson nicely demonstrated that bioorthogonal tags can be incorporated into newly synthesized DNA of both cultured cells and mouse tissues after metabolic incorporation of the tagged nucleic acid 5-ethynyl-2'-deoxyuridine [13]. An additional example is the metabolic incorporation of bioorthogonal tags into lipids in order to study protein lipidation and lipid trafficking (thoroughly reviewed in [14]). Likewise, metabolic incorporation of bioorthogonal chemical tags has been reported for proteins [15]. Kiick et al. showed the incorporation of bioorthogonal tags in the proteome of Escherichia coli (E. coli) cells upon addition of the tagged amino acid azidohomoalanine. They showed that azidohomoalanine is incorporated at sites where the amino acid methionine naturally resides. Hatzenpichler et al. showed that tagging of proteins using this abiotic amino acid is a successful approach to study newly synthesized proteins in individual microorganisms within environmental samples [9]. In addition to the proteome-wide metabolic incorporation of bioorthogonal tags, single proteins can also be modified by using amber codon suppression (reviewed in [16]). Although genetic modification is needed for this approachwith the same downsides as other genetic techniques - it is a great addition to the bioorthogonal toolkit. Attachment of abiotic tags to covalent enzyme inhibitors allows even to selective visualize active populations of enzymes in a complex mixture [17-19]. Moreover, it can be applied to the tagging of biomolecules in living multicellular organisms, such as Caenorhabditis elegans [20], zebrafish [21, 22] and mice [23].
There are now also numerous bioorthogonal reactions available for labelling these tags (thoroughly reviewed in [24] and [10]). Examples of the most often used labelling strategies are illustrated in Fig. 1c-f. The copper-catalysed Huisgen cycloaddition ( $\mathrm{ccHc}$ ) is well known for its high reaction rate and selectivity and is often used on fixed sample material, as copper is toxic to cells (Fig. 1c) [25]. The strainpromoted cycloaddition reaction is a faster alternative of the Staudinger ligation and allows in vivo labelling as there is no need of copper catalysis during this reaction (Fig. 1d) [26]. The inverse electron-demand Diels-Alder cycloaddition is a fast bioorthogonal reaction that does not require catalysis. An example of such a reaction is the cycloaddition of $s$-tetrazine and trans-cyclooctene derivatives (Fig. 1e) [27]. It is also possible to use photoactivatable chemical groups for socalled photoclick-reactions. Nitrile imine mediated [1,3]-dipolar cycloaddition reaction and has been employed to selectively functionalize an alkene genetically encoded in a protein inside $E$. coli cells [28]. The reaction procedure was reported to be simple, straightforward and non-toxic to $E$. coli cells (Fig. 1f). This variety in labelling strategies and chemical reactions highlights the versatility of the approach as it can be altered upon experimental settings. It is even possible to 'multiplex' different bioorthogonal reactions [29], i.e. to first label one class of biomolecule (in this case a proteasome subunit) using one bioorthogonal reaction and then perform an additional bioorthogonal reaction on a second class and a third reaction on a third class of bioorthogonal groups. This approach shows that the reactions are not only just bioorthogonal but also mutually orthogonal to one another.

We anticipate that bioorthogonal chemistry will be a powerful and useful addition to the CLEM-labelling toolkit. It would allow the imaging of non-protein biomolecules, and it precludes the need for genetic tagging and antibody labelling. Furthermore, the fate of biomolecules labelled by these approaches can even be monitored during the degradation process. For example, a protein labelled with bioorthogonal amino acids can be imaged, even when it is proteolytically degraded, as - unlike reporter proteins - the tags survive this catabolic pathway [30]. To the best of our knowledge, bioorthogonal reactions have not been combined with CLEM imaging up to now. This is surprising, since fluorescent imaging of bioorthogonal tags has become a commonplace over the last decade and a half [7]. In this review, we will highlight some of the inroads that have been made towards the CLEM imaging of bioorthogonal reactions.

\section{Bioorthogonal labelling for CLEM imaging}

To allow EM imaging of bioorthogonal tags, an electrondense group is required that can be introduced using a bioorthogonal reaction. The most commonly used EM- 
detectable groups are gold nanoparticles (GNPs) [31]. However, GNPs in combination with bioorthogonal labelling have not been explored for this purpose. This is remarkable, since the inverse use of bioorthogonal chemistry - to synthesize protein/DNA-modified gold particles-has been reported $[32,33]$. In these studies, no change in GNP size was observed and hydrophobic, organometallic and hydrophilic moieties could be introduced onto the particles. Brennan et al. [34] used a similar biochemical approach to produce biomoleculemodified gold particles. They first produced azide-modified gold particles and 4-pentynoic acid-modified lipase and reacted the two using a copper(II) catalyst with ascorbatereducing agent to generate the active $\mathrm{Cu}(\mathrm{I})$ species in situ. Under reducing conditions, the thiol-gold linkage appeared stable, and gold particles modified with lipase were observed. These examples indicate that GNPs have the potential to be used in combination with bioorthogonal chemistry as a labelling strategy for EM samples.

Besides these electron-dense EM-detectable particles, bioorthogonal fluorophore introduction could also be used for EM imaging; namely, in combination with CLEM. Especially considering that fluorescent bioorthogonal labelling strategies are very well established in the field. However, as stated above, to perform CLEM with this particular strategy, detectable moieties are required that are visible in both imaging modalities (e.g. fluorescent electron-dense nano or microparticles).

One approach to circumvent this would be to directly react the bioorthogonal tags with such electron-dense fluorophores. Quantum dots (Qdots) are suitable candidates for this purpose [35]. As with GNP-modification, significant development has gone into the modification of Qdots with biomolecules using bioorthogonal strategies. Again, they have not yet been used for introducing these fluorophores for CLEM. In the case of CLEM labelling, one difficulty with the modification of Qdots using 'classic' copper-catalysed Huisgen reaction (Fig. 1c) is fluorescence quenching [36]. Fluorescence quenching was circumvented by using either the strain-promoted [3+2]-cycloaddition reaction (Fig. 1d) [36] or the tetrazine-norbornene inverse electron-demand Diels-Alder reaction (Fig. 1e) [37]. In the former reaction, cyclooctyne-modified cadmium selenide/zinc sulphide (CdSe/ZnS) core-shell Qdots were modified with cyclooctyne groups and as such used to image the presence of azide-containing sugars on the surface of cultured $\mathrm{CHO}$ cells, analogous to the work performed by the Bertozzi group. However, in these experiments, no CLEM was performed. Zhang et al. [38] recently used a similar approach to image the intracellular presence of viruses. $\mathrm{CdSe} / \mathrm{ZnS}$ Qdots were modified with an azide-containing outer coating. These particles were then reacted with dibenzocyclooctyne-modified viruses that had been used to infect GFP-expressing A549 cells. This approach allowed the imaging of viral infection in these cells with good selectively. Recently, the same group published the in vivo imaging of virus infection using a near-infrared Qdot variant [39], highlighting the power of this approach.

A second alternative approach by which the subcellular location of fluorophores can be made EM-visible is by photoconversion of diaminobenzidine (DAB) [40]. This approach uses fluorophores to photooxidize $\mathrm{DAB}$, which results in precipitates after reaction with osmium. These precipitates are electron-dense and therefore EM-detectable. Such fluorophores are readily available as detecting agents for bioorthogonal reactions, and numerous examples exist of the use of these to label biomolecules. However, again, no examples have been reported of the approach where first the fluorophore is used to image a bioorthogonal label followed by photoconversion of DAB to allow for EM imaging.

A final example of an approach that has great potential for CLEM imaging of enzyme activities is the use of an aggregating probe. Ye et al. [41] reported the use of a probe that upon cleavage by the apoptosis-related caspases 3 or 7 cyclizes and precipitates to form insoluble fluorescent nanoaggregates. Rather than imaging these aggregates by CLEM, the authors imaged them by super-resolution microscopy and conventional confocal microscopy. They also showed these probes could be applied to the in vivo imaging of tumour apoptosis after treatment with doxorubicin.

\section{Conclusion}

The examples and strategies discussed here highlight the power of bioorthogonal chemistry for the labelling of biomolecules in a cellular context. To the best of our knowledge, bioorthogonal chemistry has not been explored for CLEM imaging, although many inroads have been made. We anticipate that bioorthogonal chemistry will enable CLEM imaging of molecules for which the current toolkit is not amenable, such as non-genetically template biomolecules, temporal subpopulations of proteins (those expressed in a given time window), or the imaging of enzymatically active subpopulation of a protein species.

Open Access This article is distributed under the terms of the Creative Commons Attribution 4.0 International License (http:// creativecommons.org/licenses/by/4.0/), which permits unrestricted use, distribution, and reproduction in any medium, provided you give appropriate credit to the original author(s) and the source, provide a link to the Creative Commons license, and indicate if changes were made.

\section{References}

1. van Rijnsoever C, Oorschot V, Klumperman J (2008) Correlative light-electron microscopy (CLEM) combining live-cell imaging and immunolabeling of ultrathin cryosections. Nat Methods 5(11): 973-980 
2. Ellisman $\mathrm{MH}$ et al (2012) Picking faces out of a crowd: genetic labels for identification of proteins in correlated light and electron microscopy imaging. Methods Cell Biol 111:139-155

3. Bos E et al (2014) Vitrification of Tokuyasu-style immuno-labelled sections for correlative cryo light microscopy and cryo electron tomography. J Struct Biol 186(2):273-282

4. Brown E, Verkade P (2010) The use of markers for correlative light electron microscopy. Protoplasma 244(1-4):91-97

5. Thomas CL, Maule AJ (2000) Limitations on the use of fused green fluorescent protein to investigate structure-function relationships for the cauliflower mosaic virus movement protein. J Gen Virol 81(Pt 7):1851-1855

6. Griffiths G, Lucocq JM (2014) Antibodies for immunolabeling by light and electron microscopy: not for the faint hearted. Histochem Cell Biol 142(4):347-360

7. Patterson DM, Nazarova LA, Prescher JA (2014) Finding the right (bioorthogonal) chemistry. ACS Chem Biol 9(3):592-605

8. Kolb HC, Finn MG, Sharpless KB (2001) Click chemistry: diverse chemical function from a few good reactions. Angew Chem Int Ed 40(11):2005-2021

9. Hatzenpichler R et al (2014) In situ visualization of newly synthesized proteins in environmental microbes using amino acid tagging and click chemistry. Environ Microbiol 16(8):2568-2590

10. Lang K, Chin JW (2014) Bioorthogonal reactions for labeling proteins. ACS Chem Biol 9(1):16-20

11. Saxon E, Bertozzi CR (2000) Cell surface engineering by a modified Staudinger reaction. Science 287(5460):2007-2010

12. Keppler OT et al (2001) Biochemical engineering of the $\mathrm{N}$-acyl side chain of sialic acid: biological implications. Glycobiology 11(2): $11 \mathrm{R}-18 \mathrm{R}$

13. Salic A, Mitchison TJ (2008) A chemical method for fast and sensitive detection of DNA synthesis in vivo. Proc Natl Acad Sci U S A 105(7):2415-2420

14. Hang HC, Wilson JP, Charron G (2011) Bioorthogonal chemical reporters for analyzing protein lipidation and lipid trafficking. Acc Chem Res 44(9):699-708

15. Kiick KL et al (2002) Incorporation of azides into recombinant proteins for chemoselective modification by the Staudinger ligation. Proc Natl Acad Sci U S A 99(1):19-24

16. Xie J, Schultz PG (2006) A chemical toolkit for proteins - an expanded genetic code. Nat Rev Mol Cell Biol 7(10):775-782

17. Speers AE, Adam GC, Cravatt BF (2003) Activity-based protein profiling in vivo using a copper(I)-catalyzed azide-alkyne [3+2] cycloaddition. J Am Chem Soc 125(16):4686-4687

18. Ovaa $\mathrm{H}$ et al (2003) Chemistry in living cells: detection of active proteasomes by a two-step labeling strategy. Angew Chem Int Ed 42(31):3626-3629

19. Willems LI, Overkleeft HS, van Kasteren SI (2014) Current developments in activity-based protein profiling. Bioconjug Chem 25(7): 1181-1191

20. Ullrich $\mathrm{M}$ et al (2014) Bio-orthogonal labeling as a tool to visualize and identify newly synthesized proteins in Caenorhabditis elegans. Nat Protoc 9(9):2237-2255

21. Laughlin ST et al (2008) In vivo imaging of membrane-associated glycans in developing zebrafish. Science 320(5876):664-667

22. Prescher JA, Dube DH, Bertozzi CR (2004) Chemical remodelling of cell surfaces in living animals. Nature 430(7002):873-877
23. Chang PV et al (2010) Copper-free click chemistry in living animals. Proc Natl Acad Sci U S A 107(5):1821-1826

24. Ramil CP, Lin Q (2013) Bioorthogonal chemistry: strategies and recent developments. Chem Commun 49(94):11007-11022

25. Tornøe CW, Christensen C, Meldal M (2002) Peptidotriazoles on solid phase: [1,2,3]-triazoles by regiospecific copper(I)-catalyzed 1 , 3-dipolar cycloadditions of terminal alkynes to azides. J Org Chem 67(9):3057-3064

26. Agard NJ, Prescher JA, Bertozzi CR (2004) A strain-promoted [3+ 2] azide-alkyne cycloaddition for covalent modification of biomolecules in living systems. J Am Chem Soc 126(46):15046-15047

27. Blackman ML, Royzen M, Fox JM (2008) Tetrazine ligation: fast bioconjugation based on inverse-electron-demand Diels-Alder reactivity. J Am Chem Soc 130(41):13518-13519

28. Song W et al (2008) Selective functionalization of a genetically encoded alkene-containing protein via "photoclick chemistry" in bacterial cells. J Am Chem Soc 130(30):9654-9655

29. Willems LI et al (2012) Triple bioorthogonal ligation strategy for simultaneous labeling of multiple enzymatic activities. Angew Chem Int Ed 51(18):4431-4434

30. Ouellette SP et al (2011) Chlamydia species-dependent differences in the growth requirement for lysosomes. PLoS One 6(3), e16783

31. Roth J (1996) The silver anniversary of gold: 25 years of the colloidal gold marker system for immunocytochemistry and histochemistry. Histochem Cell Biol 106(1):1-8

32. Rahim MK et al (2013) Bioorthogonal chemistries for nanomaterial conjugation and targeting. Nanotechnol Rev 2(2):215-227

33. Algar WR et al (2011) The controlled display of biomolecules on nanoparticles: a challenge suited to bioorthogonal chemistry. Bioconjug Chem 22(5):825-858

34. Brennan JL et al (2006) Bionanoconjugation via click chemistry: the creation of functional hybrids of lipases and gold nanoparticles. Bioconjug Chem 17(6):1373-1375

35. Giepmans BN et al (2005) Correlated light and electron microscopic imaging of multiple endogenous proteins using Quantum dots. Nat Methods 2(10):743-749

36. Bernardin A et al (2010) Copper-free click chemistry for highly luminescent quantum dot conjugates: application to in vivo metabolic imaging. Bioconjug Chem 21(4):583-588

37. Han H-S et al (2010) Development of a bioorthogonal and highly efficient conjugation method for quantum dots using tetrazinenorbornene cycloaddition. J Am Chem Soc 132(23):7838-7839

38. Zhang P et al (2012) Click-functionalized compact quantum dots protected by multidentate-imidazole ligands: conjugation-ready nanotags for living-virus labeling and imaging. J Am Chem Soc 134(20):8388-8391

39. Pan $\mathrm{H}$ et al (2014) Noninvasive visualization of respiratory viral infection using bioorthogonal conjugated near-infrared-emitting quantum dots. ACS Nano 8(6):5468-5477

40. Cortese K, Diaspro A, Tacchetti C (2009) Advanced correlative light/electron microscopy: current methods and new developments using Tokuyasu cryosections. J Histochem Cytochem 57(12): 1103-1112

41. Ye D et al (2014) Bioorthogonal cyclization-mediated in situ selfassembly of small-molecule probes for imaging caspase activity in vivo. Nat Chem 6(6):519-526 